\title{
POSTER
}

\section{Transformation maligne d'un lichen plan labial}

\author{
Le Guennec $B^{1}$, Dauzat $A^{1}$, Cloitre $A^{1}$, Boedec $A^{1}$, Lesclous $P^{2}$, Kimakhe $S^{3}$ \\ 1 - AHU \\ 2 - $\mathrm{PU}-\mathrm{PH}$ \\ 3 - MCU-PH ; Unité Fonctionnelle de Chirurgie Orale, Service d'Odontologie Restauratrice et Chirurgicale, CHU de \\ Nantes, Faculté de Chirurgie Dentaire de Nantes (France)
}

\section{Introduction}

Le lichen plan est une maladie inflammatoire d'origine auto immune d'évolution récidivante. Cette affection touche 1 à $2 \%$ de la population (Kuffer et coll. 2009). Toutes les régions de la muqueuse buccale peuvent être touchées sous différentes formes cliniques. Cette maladie, bénigne la plupart du temps, présenterait un taux de transformation maligne autour de $2 \%$ (Seintou et coll. 2012) presque exclusivement sous forme de carcinome épidermoide, principalement en ce qui concerne les formes érosives.

\section{Observation}

II s'agissait d'un homme de 64 ans, pris en charge pour une lésion ulcérée à la lèvre inférieure persistante depuis 4 mois. Le patient est fumeur (23 P/A) et s'expose au soleil. L'examen clinique de la lésion et de l'ensemble de la muqueuse buccale révélait des lésions kératosiques évoquant d'emblée un lichen plan buccal. L'examen histologique confirmait le diagnostic de lichen plan sans dysplasie. Le traitement associe une corticothérapie locale et l'application de vaseline ainsi que des conseils réitérés pour l'arrêt du tabac. Perdu de vue, le patient se présentait 6 mois plus tard avec une persistance de la lésion labiale inférieure sous une forme hétérogène, érosive, plus étendue et avec une base indurée. Celle-ci s'accompagnait de douleurs et d'adénopathies sous mandibulaires de consistance molle et douloureuses à la palpation. Une nouvelle biopsie confirmait la présence d'un carcinome épidermoïde. Le bilan d'extension au moyen de l'imagerie précisait un stade T1N1. Le traitement décidé était chirurgical et consistait en une résection cunéiforme de la lèvre avec exérèse complète de la lésion confirmée par l'examen anatomopathologique de la pièce opératoire.

\section{Discussion}

Le tabac et l'exposition au soleil sont des facteurs de risque dans l'apparition d'un cancer de la cavité buccale. Le lichen plan buccal présente un risque intrinsèque de transformation maligne probablement lié à l'inflammation chronique. Une association virale est parfois évoquée dans la littérature mais elle était négative dans le cas présent. Le lichen plan oral présente une grande variété de formes cliniques, le diagnostic est d'abord clinique. Un examen histologique doit être envisagé en présence de lésions érosives ou atrophiques ou de facteurs de risques associés. Une surveillance adaptée à intervalle régulier doit être envisagée pour la prise en charge précoce d'une éventuelle transformation maligne. 


\section{Conclusion}

Ce cas illustre une fois de plus le rôle du chirurgien-dentiste dans le dépistage des cancers des voies aéro-digestives supérieures et sa place dans le traitement pluridisciplinaire de ces derniers. En présence d'un lichen plan buccal ou de lésions lichénoïdes, une surveillance accrue et régulière sera entreprise notamment en présence de co-facteurs de risques tels que le tabac ou l'exposition au soleil. Le recours à la biopsie doit être envisagé en cas de doute.

\section{Lecture utile}

Seintou et coll. Histoire naturelle et transformation maligne du lichen plan buccal. 1ère partie : mise au point. Med Bucc Chir Bucc $2012 ;$ 18:89-107. 